\title{
Predictive value of gamma- glutamyltransferase for ventricular arrhythmias and cardiovascular mortality in implantable cardioverter-defibrillator patients
}

You Zhou, Shuang Zhao, Keping Chen, Wei Hua and Shu Zhang ${ }^{*}$ (i)

\begin{abstract}
Background: Gamma-glutamyltransferase (GGT) is a new predictor of cardiovascular diseases. In this study, we aimed to determine its association with ventricular arrhythmias (VAs) in implantable cardioverter-defibrillator (ICD) patients.

Methods: One hundred and forty patients implanted with ICD or cardiac resynchronization therapy defibrillator with home monitoring were studied retrospectively. The primary endpoint was appropriate ICD treatment of VAs, secondary endpoint was cardiac death.

Results: During a mean follow-up period of $44 \pm 17$ months, 78 patients (55.7\%) experienced VAs, 50 patients (35.7\%) were treated with appropriate ICD shocks and 16 patients (11.4\%) died due to cardiovascular diseases. GGT was positively correlated with high sensitivity $C$ reactive protein $(r=0.482, P<0.001)$, left ventricular end-diastolic dimension ( $r=0.175, P=0.039)$, New York Heart Association class $(r=0.199, P=0.018)$, fasting blood glucose $(r=$ $0.233, P=0.006)$ and negatively with left ventricular ejection fraction ( $r=-0.181, P=0.032)$ and high-density lipoprotein $(r=-0.313, P<0.001)$. Based on receiver operating characteristics curve, the cut-off value of $\mathrm{GGT}=56 \mathrm{U} /$ $\mathrm{L}$ was identified to predict VAs. In Kaplan-Meier survival analysis, GGT $\geq 56 \mathrm{U} / \mathrm{L}$ was associated with increased VAs $(P<0.001)$, ICD shock events $(P=0.006)$ and cardiovascular mortality $(P=0.003)$. In multivariate COX regression models, GGT $\geq 56 \mathrm{U} / \mathrm{L}$ was an independent risk factor for VAs (HR 2.253,95\%Cl:1.383-3.671, $P=0.001$ ), ICD shocks (HR 2.256, 95\%Cl:1.219-4.176, $P=0.010$ ) and cardiac death (HR 3.555, 95\%Cl:1.215-10.404, $P=0.021$ ).
\end{abstract}

Conclusions: In this ICD population, GGT $\geq 56 \mathrm{U} / \mathrm{L}$ was independently associated with VAs and cardiac death. Keywords: Gamma-glutamyltransferase, Implantable cardioverter defibrillator, Home monitoring, Ventricular arrhythmias, Cardiac death

\footnotetext{
* Correspondence: zhangshufw@163.com

State Key Laboratory of Cardiovascular Disease, Arrhythmia Center, Fuwai Hospital, National Center for Cardiovascular Diseases, Chinese Academy of Medical Sciences and Peking Union Medical College, 167 Bei Li Shi Road, Xicheng District, Beijing 100037, China
}

(c) The Author(s). 2019 Open Access This article is distributed under the terms of the Creative Commons Attribution 4.0 International License (http://creativecommons.org/licenses/by/4.0/), which permits unrestricted use, distribution, and reproduction in any medium, provided you give appropriate credit to the original author(s) and the source, provide a link to the Creative Commons license, and indicate if changes were made. The Creative Commons Public Domain Dedication waiver (http://creativecommons.org/publicdomain/zero/1.0/) applies to the data made available in this article, unless otherwise stated. 


\section{Background}

Sudden cardiac death (SCD) remains a major public health problem accounting for $50 \%$ of cardiovascular death despite advances in medical and device therapies [1]. About $80 \%$ of SCD are caused by ventricular tachycardia (VT) or ventricular fibrillation (VF) [2]. Since 1980, implantable cardioverter defibrillator (ICD) has been used to identify and terminate malignant ventricular arrhythmias (VAs) to prevent SCD. A low left ventricular ejection fraction (LVEF; < 35\%) has been used to predict SCD, but LVEF alone is not enough to fully identify the high-risk population. Many ICD patients never experienced VAs demanding ICD therapy. Therefore, it is important to identify predictors of VAs, permitting timely therapeutic interventions to improve clinical outcomes.

Home Monitoring (HM) technology in ICD patients enables continuous and instantaneous transmission of stored ICD data, which helps to timely and precisely identify the VA episodes. Previous study proved HM with automatic daily surveillance to be safe and effective [3].

Gamma-glutamyltransferase (GGT) plays a key role in the synthesis and metabolism of glutathione. Elevated GGT is a marker of antioxidant inadequacy and increased oxidative stress. Ample evidence suggested that elevated GGT was associated with increased risk of cardiovascular diseases, such as coronary heart disease, heart failure, hypertension, atrial fibrillation and SCD [4-8]. However, its impact on VAs occurrence and survival in ICD patients remains unclear. This study aimed to use ICD home monitoring feature to evaluate the predictive value of GGT for VAs and cardiac death.

\section{Methods}

\section{Study population}

A total of 140 patients who underwent ICD or cardiac resynchronization therapy defibrillator (CRT-D) implantation in Fuwai Hospital between June 2010 and June 2014 and met the inclusion criteria were enrolled in our study. Indications for device implantation in this study were defined in accordance with the published guidelines for device-based therapy [9]. Primary prevention patients were those who received ICDs or CRT-Ds on a prophylactic basis without a prior history of SCD, cardiac arrest, or sustained VT. Secondary prevention patients were those who experienced resuscitated SCD, cardiac arrest, or sustained VT before ICD implantation. The study was approved by the hospital ethics committee, and all patients provided written informed consent before entering this study.

\section{Selection criteria}

The inclusion criteria were (1) patients with ICD/CRT-D devices (Biotronik, Berlin, Germany) equipped with HM that could process daily HM transmissions, and (2) patients with GGT evaluations on admission.

\section{Demographic and clinical characteristics}

Patients' demographic characteristics including age, gender and body mass index, and baseline clinical characteristics, including echocardiographic parameters, New York Heart Association (NYHA) class, comorbidities (ischemic cardiomyopathy, hypertension, atrial fibrillation, diabetes), laboratory variables, including GGT, highsensitivity C-reactive protein (hsCRP), high density lipoprotein (HDL), low density lipoprotein (LDL), triglyceride, fasting blood glucose (FBG), and medications (betablockers, amiodarone, diuretics and angiotensinconverting enzyme inhibitors or angiotensin receptor blockers) were obtained from patients' medical records prior to device implantation.

\section{Biochemical analyses}

Venous blood samples after $12 \mathrm{~h}$ fasting were collected from each patient on the day before device implantation. All these tests were measured in the core laboratory of Fuwai Hospital by standard techniques. GGT and other biochemical parameters, including creatinine, FBG, HDL, LDL, triglyceride, were determined by Hitachi 7180 biochemistry autoanalyzer. The concentrations of hsCRP were examined using immunoturbidimetry (Beckmann Assay 360, Bera, CA, USA). The Cockcroft-Gault equation was used to estimate glomerular filtration rate.

\section{Device settings}

The ICD programmed settings were as follows: the basic pacing rate was 40-60 bpm, VT monitor zone was 140 $170 \mathrm{bpm}$, VT therapy zone was $170-210 \mathrm{bpm}$, and VF zone was over $210 \mathrm{bpm}$. All devices were programmed to provide continuous patient monitoring data.

\section{Endpoints}

The primary endpoint was the appropriate ICD therapy for VA and the secondary endpoint was cardiac death. VA was identified from the archived HM data and confirmed by intracardiac electrograms. Inappropriate events were excluded. Routine follow-ups were conducted and the status of the patient was confirmed by telephone if the patient's transmission was disrupted. If a patient died, the date and cause of death were confirmed by contacting the family.

\section{Statistical analysis}

Continuous variables were presented as the means ( \pm SDs) or medians (inter-quartile range). Student's $t$ tests and Mann-Whitney $U$ tests were used to compare normally and non-normally distributed variables, respectively. Categorical variables in each group were presented as percentages and 
were compared by the $X^{2}$ test. Spearman test was used to evaluate the correlation between GGT and other clinical or laboratory variables. Receiver operating characteristic (ROC) curve was plotted to identify a GGT cut-off value that could be used to predict VAs. The Kaplan-Meier method was used to draw the survival rate curves and the log-rank test was used to compare the differences between the curves. Multiple Cox regression analyses were performed with an enter regression model in which age, gender and each variable with a $P$ value $<0.05$ (based on the univariate analysis) were entered into the model. A P value $<0.05$ was considered statistically significant. SPSS Statistics 23.0 (SPSS, Chicago, IL, USA) and Graph Pad Prism Software 6.0 (GraphPad Software, La Jolla, CA, USA) were used to perform the statistics.

\section{Results}

Baseline characteristics

A total of 140 patients were included into this study. The mean follow-up period was $44 \pm 17$ months. Men were dominant in the study cohort (75.0\%). The average age was $56.2 \pm 13.1$ years. During the follow-ups, 78 patients $(55.7 \%)$ experienced VAs, 50 patients $(35.7 \%)$ received ICD shock therapy, and 16 patients (11.4\%) died of cardiovascular diseases.

The mean GGT on admission was $50.4 \pm 46.1 \mathrm{U} / \mathrm{L}$. ROC curve analysis determined that a GGT cut-off value of 56 $\mathrm{U} / \mathrm{L}$ could predict VA. The area under the curve was 0.635 (95\% CI:0.543-0.727, $P=0.006$ ) with a sensitivity of $43.6 \%$ and a specificity of $87.1 \%$. Comparisons of patients' baseline characteristics grouped according to the GGT cut-off value of $56 \mathrm{U} / \mathrm{L}$ were presented in Table 1. Patients whose GGT $\geq 56 \mathrm{U} / \mathrm{L}$ were more likely to be male and receive diuretics and spironolactone, with lower LVEF and higher hsCRP and FBG, than patients whose GGT $<56 \mathrm{U} / \mathrm{L}$.

The relationship between GGT and baseline variables

In correlation analysis, GGT was positively correlated with hSCRP ( $\mathrm{r}=0.482, P<0.001$ ), left ventricular end-

Table 1 Baseline characteristics according to GGT

\begin{tabular}{|c|c|c|c|c|}
\hline & Total population $(n=140)$ & GGT < 56 U/L $(n=98)$ & GGT $\geq 56 \mathrm{U} / \mathrm{L}(n=42)$ & $P$ value \\
\hline Age (years) & $56.2 \pm 13.1$ & $56.1 \pm 13.0$ & $56.5 \pm 13.4$ & 0.540 \\
\hline Male & 105 (75.0\%) & $65(66.3 \%)$ & 40 (95.2\%) & $<0.001$ \\
\hline $\mathrm{BMI}\left(\mathrm{Kg} \cdot \mathrm{m}^{-2}\right)$ & $24.2 \pm 2.4$ & $24.2 \pm 2.2$ & $24.4 \pm 2.7$ & 0.858 \\
\hline Primary prevention & $25(17.9 \%)$ & $16(16.3 \%)$ & $9(21.4 \%)$ & 0.470 \\
\hline Ischemic cardiomyopathy & $36(25.7 \%)$ & $22(22.4 \%)$ & $14(33.3 \%)$ & 0.177 \\
\hline Hypertension & $31(22.1 \%)$ & $24(24.5 \%)$ & $7(16.7 \%)$ & 0.307 \\
\hline Diabetes & $4(2.9 \%)$ & $3(3.1 \%)$ & $1(2.4 \%)$ & 0.825 \\
\hline Atrial fibrillation & $10(7.1 \%)$ & $5(5.1 \%)$ & $5(11.9 \%)$ & 0.152 \\
\hline QRS duration & $112.49 \pm 26.89$ & $111.40 \pm 26.27$ & $115.72 \pm 29.19$ & 0.559 \\
\hline LVEF (\%) & $44.9 \pm 14.7$ & $46.6 \pm 14.6$ & $40.9 \pm 14.4$ & 0.034 \\
\hline LVEDD (mm) & $57.1 \pm 13.3$ & $55.9 \pm 13.0$ & $60.0 \pm 13.7$ & 0.094 \\
\hline NYHA class I-II & $95(67.9 \%)$ & $71(72.5 \%)$ & $24(57.1 \%)$ & 0.077 \\
\hline ACEI or ARB & $74(52.9 \%)$ & 57 (58.2\%) & $17(40.5 \%)$ & 0.055 \\
\hline Beta-blocker & $122(87.1 \%)$ & $86(87.6 \%)$ & $36(85.7 \%)$ & 0.741 \\
\hline Amiodarone & $80(57.1 \%)$ & $56(57.1 \%)$ & $24(57.1 \%)$ & 1.000 \\
\hline Spironolactone & $76(54.3 \%)$ & $44(44.9 \%)$ & $32(76.2 \%)$ & 0.001 \\
\hline Digoxin & $38(27.1 \%)$ & $26(26.5 \%)$ & $12(28.6 \%)$ & 0.803 \\
\hline Diuretic & $84(60.0 \%)$ & $53(54.1 \%)$ & 31 (73.8\%) & 0.029 \\
\hline Statins & $50(35.7 \%)$ & 39 (39.8\%) & $11(26.2 \%)$ & 0.124 \\
\hline hsCRP (mg. $\left.L^{-1}\right)$ & $4.61 \pm 4.74$ & $3.58 \pm 4.06$ & $7.03 \pm 5.42$ & $<0.001$ \\
\hline eGFR $\left(\mathrm{mL} \cdot \mathrm{min}^{-1} \cdot 1.73 \mathrm{~m}^{-2}\right)$ & $80.0 \pm 24.5$ & $81.8 \pm 24.8$ & $75.8 \pm 23.5$ & 0.189 \\
\hline FBG $\left(m m o l \cdot L^{-1}\right)$ & $5.34 \pm 1.20$ & $5.14 \pm 1.00$ & $5.80 \pm 1.50$ & 0.011 \\
\hline $\mathrm{HDL}\left(\mathrm{mmol} \cdot \mathrm{L}^{-1}\right)$ & $1.03 \pm 0.36$ & $1.13 \pm 0.31$ & $1.07 \pm 0.47$ & 0.365 \\
\hline $\operatorname{LDL}\left(\mathrm{mmol} \cdot \mathrm{L}^{-1}\right)$ & $2.45 \pm 0.73$ & $2.44 \pm 0.74$ & $2.47 \pm 0.74$ & 0.849 \\
\hline Triglyceride $\left(\mathrm{mmol} \cdot \mathrm{L}^{-1}\right)$ & $1.51 \pm 0.84$ & $1.51 \pm 0.89$ & $1.53 \pm 0.74$ & 0.514 \\
\hline
\end{tabular}

Values are expressed as the mean \pm SD or $n(\%)$

Abbreviations: $B M I$ body mass index, LVEF left ventricular ejection fraction, LVEDD left ventricular end-diastolic dimension, NYHA class, New York Heart Association class, $A C E I$ or $A R B$ angiotensin-converting enzyme inhibitor or angiotensin receptor blocker, $h s C R P$ high-sensitivity C-reactive protein, eGFR estimated glomerular filtration rate, $F B G$ fasting blood glucose, $H D L$ high density lipoprotein, $L D L$ low density lipoprotein, GGT gamma-glutamyltransferase 
diastolic dimension (LVEDD; $\mathrm{r}=0.175, \quad P=0.039$ ), NYHA class $(\mathrm{r}=0.199, P=0.018)$, FBG $(\mathrm{r}=0.233, P=$ $0.006)$ and negatively with LVEF $(\mathrm{r}=-0.181, P=0.032)$ and HDL $(\mathrm{r}=-0.313, \mathrm{P}<0.001)$ (Table 2).

\section{GGT was a predictor of VAs, shock events and cardiac death}

Kaplan-Meier survival curves showed a higher incidence of VAs, shock therapy and cardiac death in patients with GGT $\geq 56 \mathrm{U} / \mathrm{L} \quad(\mathrm{P}=<0.001,0.006,0.003$, respectively; Figs. 1, 2 and 3, respectively). Adjusted by age, gender and other variables with $P$ value $<0.05$ in the univariate analysis, multivariate Cox regression analysis revealed that GGT $\geq 56 \mathrm{U} / \mathrm{L}$ was an independent predictor for VA (HR 2.253, 95\%CI:1.383-3.671, $P=0.001$; adjusted for age, gender, LVEDD, ischemic cardiomyopathy and diabetes), ICD shock therapy (HR 2.256, 95\%CI:1.2194.176, $P=0.010$; adjusted for age, gender, LVEDD and ischemic cardiomyopathy) and cardiac death (HR 3.555, 95\%CI:1.215-10.404, $P=0.021$; adjusted for age, gender, LVEDD, LVEF and diabetes) (Table 3).

\section{Discussion}

This study showed that GGT $\geq 56 \mathrm{U} / \mathrm{L}$ was an independent risk factor for VA, ICD shock therapy and cardiovascular death in ICD patients. GGT is a key enzyme in the extracellular metabolism of glutathione and is considered as a marker of oxidative stress and inflammation [10]. GGT may also be directly involved in the pathophysiology of atherosclerosis in view of the presence of catalytically active GGT in atherosclerotic plaques and a correlation between GGT activity and plaque instability $[11,12]$. Many studies demonstrated that elevated GGT was associated with increased risk of cardiovascular diseases. A meta-analysis including over 1.23 million subjects showed a positive association in a log-linear

Table 2 Correlation analysis between GGT and Baseline Variables

\begin{tabular}{lll}
\hline Baseline Variables & $r$ & $P$-value \\
\hline Body mass index & 0.094 & 0.273 \\
QRS duration & 0.191 & 0.111 \\
Left ventricular ejection fraction & -0.181 & 0.032 \\
Left ventricular end-diastolic dimension & 0.175 & 0.039 \\
New York Heart Association class & 0.199 & 0.018 \\
High-sensitivity C-reactive protein & 0.482 & $<0.001$ \\
Estimated glomerular filtration rate & -0.140 & 0.099 \\
Fasting blood glucose & 0.233 & 0.006 \\
High density lipoprotein & -0.313 & $<0.001$ \\
Low density lipoprotein & 0.035 & 0.682 \\
Triglyceride & 0.100 & 0.241 \\
\hline
\end{tabular}

Abbreviation: GGT gamma-glutamyltransferase fashion between elevated GGT and cardiovascular diseases $(\mathrm{RR}=1.23[1.16-1.29] ; P<0.001)$ [4]. Poelzl et al.'s study demonstrated that GGT was associated with disease severity and an independent predictor of death or heart transplantation in patients with chronic heart failure [5]. Some studies also supported that elevated GGT was associated with VA. A cohort study of 1780 Finnish men demonstrated that GGT activity was positively and log-linearly associated with future risk of VA and SCD $[8,13]$. Elevated GGT was also found to be an independent risk factor for VT in patients with type 2 diabetes [14]. However, these studies focused on community population or patients without high SCD risk. The patients included in our study were at high risk for SCD and most had structural heart disease with decreased LVEF. GGT could predict life-threatening VA in this population. On the other hand, racial disparities may also affect the predictive power of GGT [15]. This study proved predictive value of GGT in Asian population with high SCD risk.

Oxidative stress and inflammation may play a role between GGT and arrhythmogenesis. Oxidative stress can lead to VA or SCD by inducing myocardial ischemia, remodeling of ion channels and affecting autonomic nervous system function [16]. Parajuli et al. [17] found that, in the heart specimens of patients with non-ischemic cardiomyopathy, patients with VA than those without VA had a reduction in the ratio of glutathione and oxidized glutathione with an increase in NADPH oxidase activity, accompanied by increased TRPM7 expression and Connexin 43 redistribution. Inflammatory cytokines such as tumor necrosis factor $-\alpha$ and interleukin -6 had also been found involved in the development of VA by modulating ion channels [18]. Anti-inflammatory therapy was proved to reduce spontaneous and inducible VAs in murine models [19]. Our study found a moderate positive correlation between GGT and hsCRP, and patients in GGT $\geq 56 \mathrm{U} / \mathrm{L}$ group had significantly higher hsCRP, indicating elevated GGT was associated with systemic inflammation. Lee et al. [20] also found that GGT and CRP were positively correlated and GGT could be raised before elevation of serum CRP concentration. Oxidative stress consumes glutathione, so elevated GGT may be its compensatory response. Oxidative stress is also closely related to carbohydrate and lipid metabolism. In the present study, GGT was correlated with FBG and HDL, and diabetes mellitus was also found to be an independent predictor of VA. In patients with new-onset diabetes and pre-diabetes, elevated GGT was associated with prolonged QT interval and increased QT dispersion [21]. Elevated GGT is associated with oxidative stress and systemic inflammation, thus reflecting electrophysiological instability. Compared with GGT < 56 U/L group, patients with GGT $\geq 56 \mathrm{U} / \mathrm{L}$ had lower LVEF and 


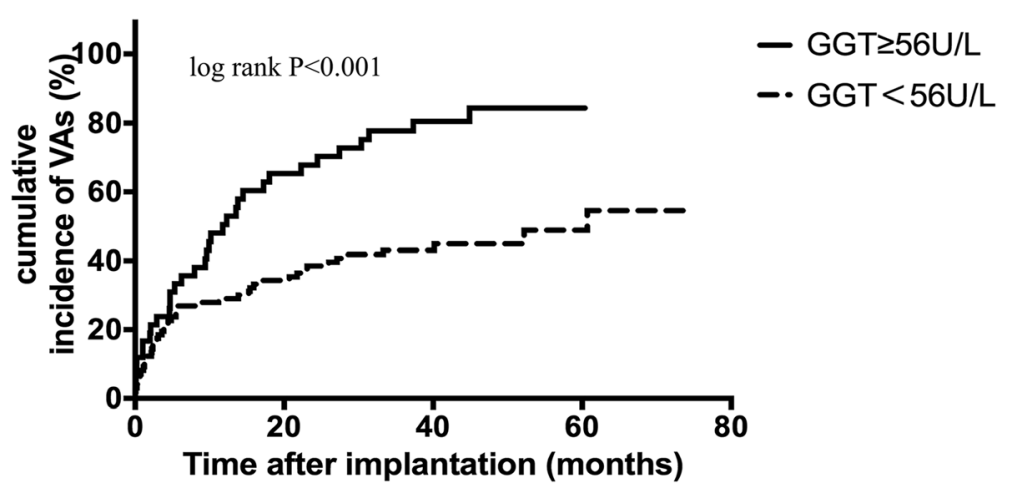

Fig. 1 Kaplan-Meier estimates of the cumulative incidence of VAs (log rank $P<0.001$ ). Abbreviations: VAs, ventricular arrhythmias

more patients used diuretics and spironolactone to treat heart failure. The correlation analysis also showed that GGT was negatively correlated with LVEF and positively correlated with LVEDD and NYHA class. The possible reason might be hepatic congestion caused by left ventricular dysfunction, and antioxidant inadequacy and increased oxidative stress, which caused elevated GGT and involved in ventricular remodeling. The association between GGT and NYHA class, LVEF and LVEDD were also found in previous studies $[4,22]$. Patients with more advanced heart failure might need diuretics and spironolactone to further control syndrome. In Poelzl et al.'s study, heart failure patients with elevated GGT were also more on diuretics and spironolactone [5]. Thus, heart function might also play a role in GGT for predicting VA.

The association between GGT and cardiac death had been found in a large number of studies. Framingham offspring study found that GGT could be used as a marker for metabolic syndrome and cardiovascular diseases and predict the risk of death [23]. In a 17-year follow-up, an Austrian cohort study of 163,944 adults revealed higher GGT was significantly associated with cardiovascular mortality, showing a clear dose-response relationship [24]. A meta-analysis by Wang et al. [25] also confirmed a positive correlation between GGT and the risk of cardiovascular death. In our study, GGT $\geq 56 \mathrm{U} / \mathrm{L}$ was associated with 3.5-fold risk of cardiovascular death in ICD patients, which proved GGT's predictive value in ICD patients. GGT might be helpful in risk stratification in this population.

\section{Study limitations}

This retrospective single-center study had a relatively small sample size and included a mixture of patients receiving ICDs or CRT-Ds for primary or secondary prevention. Predictive values of GGT could decrease in female or the elderly $[26,27]$. However, further subgroup analysis according to gender or age did not make sense for statistical reasons. We also did not measure GGT during follow-up to observe its change. Therefore, a prospective study that includes adequate number of patients is necessary to confirm the prognostic value of GGT. Secondly, racial disparities also affected the predictive power of GGT [15]. This study only included Chinese patients. The results may not apply to other ethnic populations. Finally, only one inflammatory biomarker (hsCRP) was analyzed and compared to GGT. Other inflammatory cytokines such as tumor necrosis factor $-\alpha$

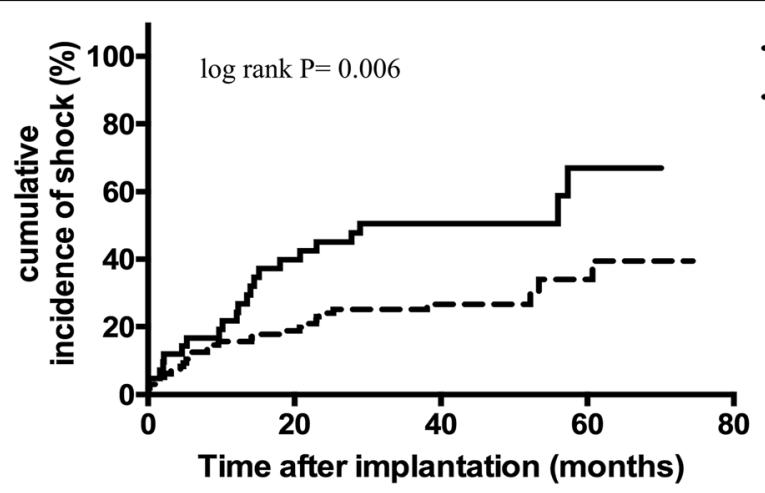

Fig. 2 Kaplan-Meier estimates of the cumulative incidence of shocks (log rank $P=0.006$ ) 


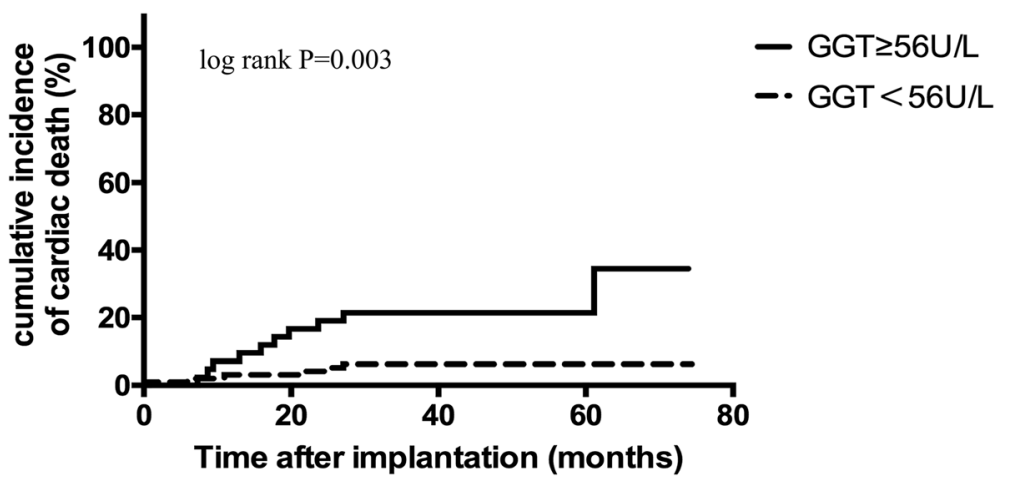

Fig. 3 Kaplan-Meier estimates of the cumulative incidence of cardiac death (log rank $P=0.003$ )

and interleukin -6 were not available in this retrospective study.

\section{Conclusions}

To conclude, elevated GGT is associated with VAs and cardiac death in ICD patients. GGT measurements may have the potential to improve patient selection for ICD therapy. Further studies are warranted to verify our findings.

Table 3 Univariate and multivariate Cox analysis for endpoints

\begin{tabular}{llll}
\hline & $H R$ & $95 \% \mathrm{Cl}$ & $P$ value \\
\hline $\begin{array}{l}\text { univariate analysis } \\
\text { VAs }\end{array}$ & & & \\
GGT $\geq 56 \mathrm{U} / \mathrm{L}$ & 2.429 & $1.542-3.826$ & $<0.001$ \\
shock & & & \\
GGT $\geq 56 \mathrm{U} / \mathrm{L}$ & 2.161 & $1.234-3.784$ & 0.007 \\
cardiac death & & & \\
GGT $\geq 56 \mathrm{U} / \mathrm{L}$ & 4.171 & $1.515-11.483$ & 0.006
\end{tabular}

multivariate analysis

\begin{tabular}{llll}
$V_{\text {VAs }}{ }^{\mathrm{a}}$ & & & \\
GGT $\geq 56 \mathrm{U} / \mathrm{L}$ & 2.253 & $1.383-3.671$ & 0.001 \\
$\begin{array}{l}\text { Diabetes } \\
\text { shock }\end{array}$ & 3.168 & $1.082-9.276$ & 0.035 \\
GGT $\geq 56 \mathrm{U} / \mathrm{L}$ & 2.256 & $1.219-4.176$ & 0.010 \\
LVEDD & 1.026 & $1.003-1.050$ & 0.029 \\
cardiac death & & & \\
GGT & & & \\
Diabetes & 3.555 & $1.215-10.404$ & 0.021 \\
\hline
\end{tabular}

a adjusted for age, gender, LVEDD, ischemic cardiomyopathy, diabetes, GGT $\geq 56 \mathrm{U} / \mathrm{L}$

$\mathrm{b}$ adjusted for age, gender, LVEDD, ischemic cardiomyopathy, GGT $\geq 56 \mathrm{U} / \mathrm{L}$ c adjusted for age, gender, LVEDD, left ventricular ejection fraction, diabetes, GGT $\geq 56 \mathrm{U} / \mathrm{L}$

Abbreviations: VAs ventricular arrhythmias, $H R$ hazard ratio, $\mathrm{Cl}$ confidence interval, GGT gamma-glutamyltransferase, LVEDD left ventricular end-diastolic diameter

\section{Abbreviations}

CRT-D: Cardiac resynchronization therapy defibrillator; FBG: Fasting blood glucose; GGT: Gamma-glutamyltransferase; HDL: High density lipoprotein; HM: Home monitoring; hsCRP: High-sensitivity C-reactive protein

ICD: Implantable cardioverter defibrillator; LDL: Low density lipoprotein LVEDD: Left ventricular end-diastolic dimension; LVEF: Left ventricular ejection fraction; NYHA: New York heart association; ROC: Receiver operating characteristic; SCD: Sudden cardiac death; VA: Ventricular arrhythmias;

VF: Ventricular fibrillation; VT: Ventricular tachycardia

\section{Acknowledgements}

None.

\section{Authors' contributions}

YZ, SZ1 and SZ2 contributed to the conception or design of the work. YZ SZ1, KPC and WH contributed to the acquisition, analysis, or interpretation of data for the work. YZ and SZ1 drafted the manuscript. SZ2 critically revised the manuscript. All gave final approval and agree to be accountable for all aspects of work ensuring integrity and accuracy.

Funding

None.

Availability of data and materials

The datasets generated and analysed during the current study are not publicly available due to the Fuwai Hospital regulations, but are available from the corresponding author on reasonable request.

Ethics approval and consent to participate

The study was approved by the Ethics Committee of Fuwai Hospital before the start of the work, and each patient signed written consent form.

\section{Consent for publication}

Not applicable.

Competing interests

The authors declare that they have no competing interests.

Received: 24 December 2018 Accepted: 21 May 2019

Published online: 30 May 2019

\section{References}

1. Myerburg RJ, Junttila MJ. Sudden cardiac death caused by coronary heart disease. Circulation. 2012:125:1043-52.

2. Josephson M, Wellens HJ. Implantable defibrillators and sudden cardiac death. Circulation. 2004;109:2685-91.

3. Varma $N$, Epstein AE, Irimpen A, Schweikert R, Love C, Investigators TRUST. Efficacy and safety of automatic remote monitoring for implantable cardioverter-defibrillator follow-up. Circulation. 2010;122:325-32. 
4. Kunutsor SK, Apekey TA, Khan H. Liver enzymes and risk of cardiovascular disease in the general population: a meta-analysis of prospective cohort studies. Atherosclerosis. 2014;236:7-17.

5. Poelzl G, Eberl C, Achrainer H, Doerler J, Pachinger O, Frick M, et al. Prevalence and prognostic significance of elevated gammaglutamyltransferase in chronic heart failure. Circ Heart Fail. 2009:2:294-302.

6. Liu CF, Gu YT, Wang HY, Fang NY. Gamma-Glutamyltransferase level and risk of hypertension: a systematic review and meta-analysis. PLoS One. 2012;7:e48878.

7. Tekin G, Tekin YK, Senarslan DA, Gocmen AY, Senarslan O, Erbay AR. Serum Y-glutamyltransferase activity in patients with nonvalvular atrial fibrillation. Angiology. 2013;64:157-60.

8. Kunutsor SK, Hassan K, Laukkanen JA. Y-Glutamyltransferase and risk of sudden cardiac death in middle-aged Finnish men: a new prospective cohort study. J Am Heart Assoc 2016; 220:718-725.

9. Epstein AE, DiMarco JP, Ellenbogen KA, Estes NA 3rd, Freedman RA, Gettes LS, et al. ACC/AHA/HRS 2008 guidelines for device-based therapy of cardiac rhythm abnormalities: a report of the American College of Cardiology/ American Heart Association task force on practice guidelines (writing committee to revise the ACC/AHA/NASPE 2002 guideline update for implantation of cardiac pacemakers and Antiarrhythmia devices): developed in collaboration with the American Association for Thoracic Surgery and Society of Thoracic Surgeons. Circulation. 2008;117:e350-408.

10. Yamada J, Tomiyama H, Yambe M, Koji Y, Motobe K, Shiina K, et al. Elevated serum levels of alanine aminotransferase and gamma glutamyltransferase are markers of inflammation and oxidative stress independent of the metabolic syndrome. Atherosclerosis. 2006;189:198-205.

11. Pucci A, Franzini M, Matteucci M, Ceragioli S, Marconi M, Ferrari M, et al. Bgamma-glutamyltransferase activity in human vulnerable carotid plaques. Atherosclerosis. 2014;237:307-13.

12. Paolicchi A, Emdin M, Ghliozeni E, Ciancia E, Passino C, Popoff G, et al. Human atherosclerotic plaques contain gamma-Glutamyl Transpeptidase enzyme activity. Circulation. 109:1440.

13. Kunutsor SK, Laukkanen JA, Bluemke DA, Butler J, Khan H. Baseline and long-term gamma-glutamyltransferase, heart failure and cardiac arrhythmias in middle-aged Finnish men: prospective study and pooled analysis of published evidence. Eur J Prev Cardiol. 2016;23:1354-62.

14. Mantovani A, Rigamonti A, Bonapace S, Bolzan B, Pernigo M, Morani G, et al. Nonalcoholic fatty liver disease is associated with ventricular arrhythmias in patients with type 2 diabetes referred for clinically indicated 24-h holter monitoring. Diabetes Care. 2016;39:1416-23.

15. Du G, Song Z, Qin Z. Gamma-glutamyltransferase is associated with cardiovascular and all-cause mortality: a meta-analysis of prospective cohort studies. Prev Med. 2013;57:31-7.

16. Yang KC, Kyle JW, Makielski JC, Dudley SC Jr. Mechanisms of sudden cardiaC death: oxidants and metabolism. Circ Res 2015; 116:1937-1955.

17. Parajuli N, Valtuille L, Basu R, Famulski KS, Halloran PF, Sergi C, et al. Determinants of ventricular arrhythmias in human explanted hearts with dilated cardiomyopathy. Eur J Clin Investig. 2016;45:1286-96.

18. Francis Stuart SD, De Jesus NM, Lindsey ML, Ripplinger CM. The crossroads of inflammation, fibrosis, and arrhythmia following myocardial infarction. J Mol Cell Cardiol. 2015;91:114-22.

19. De Jesus NM, Wang L, Lai J, Rigor RR, Francis Stuart SD, Bers DM, et al. Antiarrhythmic effects of interleukin 1 inhibition after myocardial infarction. Heart Rhythm. 2017;14:727-36.

20. Lee DH, Jacobs DR Jr. Association between serum gamma-glutamyltransferase and C-reactive protein. Atherosclerosis. 2005; 178:327-330.

21. Wang K, Li L, Wu Y, Yang Y, Chen J, Zhang D, et al. Increased serum gamma-glutamyltransferase levels are associated with ventricular instability in type 2 diabetes. Endocrine. 2016;52:63-72.

22. Radovanovic S, Savic-Radojevic A, Pekmezovic T, Markovic O, Memon L, Jelic S, et al. Rev Esp Cardiol. 2014;67:632-42.

23. Lee DS, Evans JC, Robins SJ, Wilson PW, Albano I, Fox CS, et al. Gamma Glutamyl transferase and metabolic syndrome, cardiovascular disease, and mortality risk the Framingham heart study. Arterioscler Thromb Vasc Biol. 2007;27:127-33.

24. Ruttmann E, Brant LJ, Concin H, Diem G, Rapp K, Ulmer H, et al. $\gamma$ Glutamyltransferase as a risk factor for cardiovascular disease mortality. Circulation. 2005;112:2130-7.

25. Wang J, Zhang D, Huang R, Li X, Huang W. Gamma-glutamyltransferase and risk of cardiovascular mortality: a dose-response meta-analysis of prospective cohort studies. PLoS One. 2017;12:e0172631.
26. Lee DH, Buijsse B, Steffen L, Holtzman J, Luepker R, Jacobs DR Jr. Association between serum gamma-glutamyltransferase and cardiovascular mortality varies by age: the Minnesota heart survey. Eur J Cardiovasc Prev Rehabil 2009: 16:16-20.

27. Lee DH, Silventoinen $K, H u$ G, Jacobs DR Jr, Jousilahti P, Sundvall J, et al. Serum gamma-glutamyltransferase predicts non-fatal myocardial infarction and fatal coronary heart disease among 28,838 middle-aged men and women. Eur Heart J. 2006;27:2170-6.

\section{Publisher's Note}

Springer Nature remains neutral with regard to jurisdictional claims in published maps and institutional affiliations.
Ready to submit your research? Choose BMC and benefit from:

- fast, convenient online submission

- thorough peer review by experienced researchers in your field

- rapid publication on acceptance

- support for research data, including large and complex data types

- gold Open Access which fosters wider collaboration and increased citations

- maximum visibility for your research: over $100 \mathrm{M}$ website views per year

At $\mathrm{BMC}$, research is always in progress.

Learn more biomedcentral.com/submissions 